(c)Auteure. Cette œuvre, disponible à http://dx.doi.org/10.18162/fp.2020.700, est distribuée sous licence Creative Commons Attribution 4.0 International http://creativecommons.org/licences/by/4.0/deed.fr

\title{
Avantages et contraintes de la formation à distance des acteurs de la gouvernance scolaire régionale en Côte d'Ivoire
}

\begin{abstract}
Marie-Florentine Ahou Aye
Institut Pédagogique National de l'Enseignement Technique et Professionnel - IPNETP
\end{abstract}

(Côte d'Ivoire)

\author{
Benefits and constraints of remote learning \\ for regional education authorities in Ivory Coast
}

doi: $10.18162 / f p .2020 .700$

\section{ésumé}

Cette étude analyse les approbations, appréhensions et suggestions des directeurs régionaux et départementaux de l'Enseignement technique, quant à l'exploitation de la formation à distance comme solution à la discontinuité du renforcement de leurs capacités. Le contexte de pandémie qui prévaut actuellement constitue un environnement facilitant pour la mise en œuvre de cette pratique pédagogique. Léchantillon se compose de 12 directeurs régionaux et départementaux, élargi aux enseignants responsables de leur formation continue. II ressort de l'analyse des résultats une large adhésion aux enseignements en ligne, malgré quelques appréhensions exprimées. Toutefois, le faible niveau d'équipement des organisations scolaires et l'usage limité des technologies éducatives constituent des contraintes.

\section{Mots-clés}

Renforcement des capacités; formation à distance; avantages; contrainte ; représentations des acteurs scolaires. Contexte de pandémie.

\begin{abstract}
This study examines the adoptions, misgivings, and suggestions of regional and departmental authorities for vocational education concerning the use of remote learning to prevent discontinuity of skills acquisition. The sample comprised 12 regional and departmental directors and the instructors who delivered their continuous education programs. The analysis results show substantial participation in online learning despite some expressed apprehension. However, a lack of equipment at the schools and a limited use of educational technologies acted as constraints.
\end{abstract}

Keywords

Capacity-building, remote learning, benefits, constraints, educators' perceptions.

\section{Introduction}

Lobjectif de ce texte est de présenter les résultats d'une enquête menée auprès de différents acteurs de l'Enseignement Technique en Côte d'Ivoire, afin de comprendre leur utilisation des TIC dans le contexte de la pandémie. Le service de l'Enseignement en Côte d'Ivoire comprend trois degrés d'enseignement: le degré de l'Enseignement préscolaire et primaire; le degré de l'Enseignement secondaire et le degré de l'Enseignement supérieur. Chaque degré d'enseignement comporte des cycles d'apprentissage ayant des objectifs, des contenus, une organisation pédagogique et des modalités d'évaluation des acquis qui leur sont spécifiques. Lenseignement technique ivoirien est une partie intégrante de l'Enseignement Secondaire. Selon la loi n ${ }^{\circ}$ 95-696 du 7 septembre 1995 relative à l'Enseignement, en son article 34b, l'Enseignement secondaire comprend des filières spécialisées organisée en cycles : les filières de l'Enseignement général et technique et les filières de l'Enseignement professionnel. Les niveaux hiérarchiques dans l'organisation du Ministère responsable de l'Éducation Nationale et de l'Enseignement Technique (MENET) se présentent comme suit : au niveau central: le ministre et son cabinet, les structures spécialisées rattachées au cabinet ministériel et les directions centrales; au niveau régional et départemental: les directions régionales et les directions départementales et au niveau local: les lycées, collèges et établissements d'enseignement primaire et préscolaire. Les directions régionales et départementales de l'Éducation Nationale (Enseignement Général et Enseignement Technique) font la jonction entre le niveau hiérarchique central où se définissent les politiques et stratégies éducatives et les établissements scolaires au niveau desquels ces stratégies sont traduites localement en projets. La direction régionale ou 
départementale occupe ainsi une position charnière entre les instances hiérarchiques centrales et les instances hiérarchiques locales. Ce sont des structures déconcentrées de l'éducation. En Côte d'Ivoire, selon les résultats d'un sondage réalisé en 2019 par l'intermédiaire d'un cabinet d'études statistiques et d'après les résultats de l'état des lieux réalisé dans le cadre de l'élaboration du plan stratégique de réforme de l'Enseignement technique 2012-2021, le renforcement des capacités de ces acteurs scolaires est effectué de façon irrégulière. En effet, cette formation se fait à un rythme moyen d'une (1) formation tous les 5 ans pour $50 \%$ des répondants du sondage et tous les 3 ans pour 33,33\% d'entre eux. La durée moyenne de formation est en général d'une (1) semaine.

Ce renforcement des capacités est assuré par les enseignants des écoles supérieures de formation des personnels de l'éducation. Ce sont des institutions d'éducation qui relèvent de l'Enseignement Supérieur et qui forment les professeurs des lycées et collèges. Elles font partie des Grandes Écoles publiques de formation qui ont le statut d'Établissements Publics Nationaux (EPN). Elles sont dotées d'une personnalité morale et d'une autonomie de gestion, à l'instar des Universités publiques. Ces institutions scolaires chargées d'assurer le renforcement des capacités des directeurs régionaux sont implantées uniquement dans la capitale économique du pays, à Abidjan, tandis que les directions régionales et départementales de l'Éducation sont réparties sur l'ensemble du territoire national, dans le but de rapprocher l'administration des administrés. Ce contexte expose la difficulté d'assurer une formation continue à un rythme régulier à ces acteurs de l'éducation. Aussi la mise en ligne des cours constitue-t-elle, à notre sens, une pratique pédagogique à exploiter davantage dans le secteur de l'éducation/formation en Côte d'Ivoire. Deschênes, Bilodeau, Bourdages, Dionne, Gagné, Lebel et Rada-Donat (1996, pp 9-21) définissent l'enseignement en ligne comme une pratique éducative privilégiant une démarche d'apprentissage qui rapproche le savoir de l'apprenant. L'apprentissage se fait donc à l'issue de l'interaction entre l'apprenant et l'objet d'apprentissage. Deschênes et Lebel (1994) et Piaget (1975) cité par Legendre-Bergeron (1980) ont défini cinq caractéristiques de la démarche utilisée dans la formation à distance pour réaliser l'apprentissage : l'accessibilité, la contextualisation, la flexibilité, la diversification des interactions et la désaffectivation des savoirs. Au sujet de l'accessibilité du savoir, Deschênes et Maltais (2006), citant Jacquinot (1993), expliquent que la formation à distance permet d'apprendre en proposant des situations d'enseignement-apprentissage qui tiennent compte des contraintes individuelles de chaque apprenant, c'est-à-dire des distances spatiale, temporelle, technologique, psychosociale et socio-économique. En ce qui concerne son caractère flexible, ces auteurs parlent de la formation à distance comme d'une pratique éducative qui utilise des approches permettant à l'étudiant de planifier dans le temps et dans l'espace ses activités d'étude et son rythme d'apprentissage. Ils ajoutent qu'elle permet de concevoir des activités offrant à l'apprenant des choix dans les contenus, dans les méthodes et dans les interactions et de prendre ainsi en compte les caractéristiques individuelles de chaque apprenant. Ainsi, elle facilite l'intégration des savoirs scientifiques aux savoirs pratiques et donc, le transfert des connaissances. En somme, la formation à distance, en plus de faciliter l'accessibilité des savoirs et d'offrir des possibilités de diversification des interactions et des activités, est également une voie de développement de l'éducation inclusive, puisquelle prend en compte les spécificités des apprenants. 


\section{Problématique et cadre théorique}

Les enseignements en ligne pour le renforcement des capacités des leaders scolaires au niveau hiérarchique régional constituent un enjeu de performance du système éducatif, en ce sens que la direction régionale ou départementale occupe une position charnière entre les instances hiérarchiques centrales et les instances hiérarchiques locales. Une discontinuité du rythme du renforcement des capacités des directeurs régionaux et départementaux de l'éducation est donc un facteur de dysfonctionnement des structures éducatives à l'échelon régional et, par conséquent, du système éducatif du fait de l'interdépendance des composantes de tout système. Cette étude établit ainsi une relation entre la performance organisationnelle des structures scolaires et la modification des représentations des acteurs scolaires par l'intégration de nouvelles pratiques éducatives. Jodelet (2006) explique à ce sujet que le changement des représentations des acteurs signifie une nouvelle façon d'appréhender son environnement et d'agir sur lui. De même, pour Bedin et Fournier citées par Grahay (2014), la représentation que l'on se fait d'une situation contribue à construire cette situation. Selon cette perspective théorique, la qualité de la gouvernance scolaire à l'échelon régional passera par l'acceptation de la formation à distance comme un complément de la formation en mode présentiel des directeurs régionaux et départementaux de l'Éducation. D'où l'intérêt, dans cette étude, d'impliquer les futurs bénéficiaires et animateurs des cours en ligne, dans la réflexion pour la planification de leur mise en œuvre. Notre recherche sera orientée en fonction des interrogations suivantes : Quelle analyse doit-on effectuer au sujet des avantages et contraintes de la formation à distance, à partir des approbations, appréhensions et suggestions des directeurs régionaux et départementaux de l'Enseignement technique ? Comment les futurs bénéficiaires appréhendent-ils la formation à distance, comme mode de renforcement de leurs capacités ? Et quelles sont les conditions matérielles de réalisation de cette innovation pédagogique?

Cette étude vise ainsi à analyser les avantages et les contraintes de la formation à distance des directeurs régionaux et départementaux de l'Enseignement technique en Côte d'Ivoire, à travers leurs représentations en matière d'approbations, d'appréhensions et de suggestions, ainsi que celles des enseignants chargés du renforcement de leurs capacités.

\section{Méthodologie}

La population de l'étude concerne principalement les acteurs de la gouvernance scolaire régionale. L'échantillon se compose de 12 directeurs régionaux et départementaux. Un premier prélèvement a été effectué par choix raisonné et par souci de représentativité territoriale, sur une population totale de 46 individus (effectif de 2018), soit 10 à Abidjan et dans les localités périphériques et 13 dans les villes de l'intérieur du pays. Ensuite, sur ce sous-total de 23 individus, un deuxième prélèvement a été fait, par choix aléatoire, pour retenir aussi la moitié de cette sous-population : soit 5 à Abidjan et périphérie et 7 dans les localités urbaines de l'intérieur, ce qui donne 12 répondants prélevés en fin de compte (soit $26 \%$ de la population totale). Par ailleurs, la population de l'étude a été élargie aux enseignants responsables de leur formation continue. Ce deuxième échantillon d'acteurs scolaires a été choisi sur la base de la disponibilité des enseignants à la période des enquêtes, soit un prélèvement également de 12 individus sur 18 (67\%). Le questionnaire a été l'instrument de collecte utilisé, avec de 
nombreuses questions ouvertes, afin de permettre aux répondants de livrer, de manière semi-directive, leurs perceptions et suggestions sur la formation à distance, comme mode de renforcement de leurs capacités. Dans une démarche ethnométhodologique, les répondants ont été placés dans une situation où ils devaient exprimer leurs perceptions de la formation à distance et contribuer à la réflexion sur la planification de sa mise en œuvre. L'approche de recherche qualitative de Miles et Huberman (2003) a été choisie au regard de la nature non métrique des données recueillies. L'analyse a été réalisée à partir du logiciel Sphinx version $5^{1}$ qui possède des fonctionnalités pour traiter à la fois des données quantitatives et des données qualitatives.

L'analyse des données recueillies a été menée suivant deux grands axes : d'une part, la signification de la formation à distance pour les acteurs en présence et d'autre part, les conditions de réalisation des enseignements en ligne qui s'avèrent être des contraintes.

La signification que revêt la formation à distance pour les répondants est révélée à travers le niveau d'adhésion exprimée à l'égard de cette pratique pédagogique; la reconnaissance des avantages de l'usage des technologies éducatives avec l'exemple du PowerPoint et les appréhensions et réserves exprimées.

Quant aux conditions de réalisation des enseignements en ligne, elles sont analysées sous deux angles: le niveau d'usage des TIC par les acteurs scolaires en présence et le niveau d'équipement des structures éducatives concernées.

\section{Résultats et analyse}

\section{Renforcement des capacités à distance : quelle signification pour les acteurs en présence?}

La formation à distance comme alternative à la formation en mode présentiel est perçue diversement. L'analyse des approbations et appréhensions vis-à-vis des cours en ligne permet d'ouvrir une lucarne sur la signification que revêt cette innovation pédagogique pour les acteurs scolaires.

\section{Analyse des approbations exprimées vis-à-vis de la formation à distance}

Lorsqu'il a été demandé aux directeurs régionaux et départementaux de l'Enseignement technique de dire s'ils pensaient posséder toutes les qualifications nécessaires pour exécuter avec efficacité leur mission, 10 sur les 12 interrogés, soit $83 \%$, ont répondu non. Cette réponse exprime la prise de conscience par la majorité de la population cible de ses besoins de formation en gouvernance scolaire. En conséquence, la formation à distance comme mode de renforcement de leurs capacités a été approuvée à $75 \%$ (09 cas sur 12) (figure 1). 


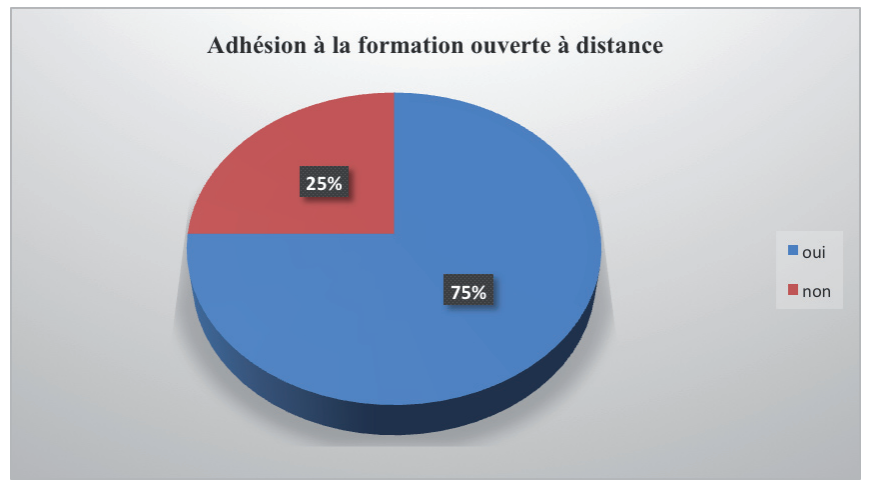

Figure 1

Adhésion à la formation ouverte à distance (FOAD)

Au titre des raisons avancées pour justifier leur approbation à l'égard de la formation à distance (figure 2), la majorité des répondants (soit $60 \%$ ) estiment que cette pratique pédagogique leur permettra d'avoir des mises à jour de façon régulière de leurs connaissances et pratiques professionnelles. La formation en ligne est donc perçue comme une alternative intéressante à la formation continue classique en mode présentiel. Ensuite, la suppression des frais annexes de la formation (coûts de transports, d'hébergement et de restauration) occupe la deuxième place des raisons en faveur de la formation à distance, avec un suffrage de $40 \%$. La troisième raison notable, qui totalise un suffrage de $30 \%$, est le gain de temps par le fait de ne plus avoir à se déplacer pour se former. De l'analyse des raisons avancées par les directeurs régionaux pour expliquer leur adhésion à la formation à distance, il ressort donc la trilogie : mise à jour des connaissances et des pratiques de gestion, annulation des dépenses liées au transport, à l'hébergement et à la restauration et, enfin, le gain de temps.

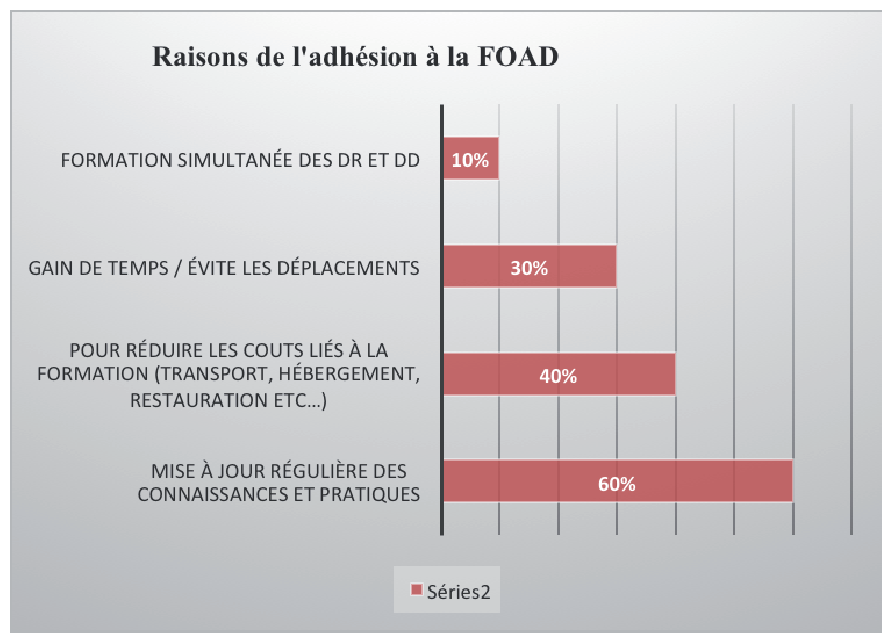

Figure 2

Avantages de la formation à distance selon les directeurs régionaux 
Quant aux points de vue des enseignants, ils ne sont pas très différents de ceux des directeurs régionaux de l'éducation : les trois points saillants de leurs réponses sont l'annulation des dépenses liées au voyage, à l'hébergement et à la restauration ; l'enrichissement des capacités par l'accessibilité des formations en ligne et le gain de temps (tableau 1).

\section{Tableau 1}

Avantages de la formation à distance selon les enseignants

\begin{tabular}{l|l|l}
\hline Principales idées avancées & $\begin{array}{l}\text { Suffrages } \\
\text { exprimés }\end{array}$ & $\begin{array}{l}\text { Proportions } \\
(\%)\end{array}$ \\
\hline $\begin{array}{l}\text { Économie en matière d'annulation des dépenses liées au voyage, à l'hébergement } \\
\text { et à la restauration. }\end{array}$ & 6 & 33,33 \\
\hline Enrichissement des capacités par l'accessibilité des formations en ligne & 6 & 33,33 \\
\hline Gain de temps / Évite de se déplacer & 3 & 16,66 \\
\hline Facilite l'accès aux cours pour les apprenants, quel que soit le lieu & 2 & 11,11 \\
\hline Bon outil de formation & 1 & 5,55 \\
\hline Total & $\mathbf{1 8}$ & $\mathbf{1 0 0}$ \\
\hline
\end{tabular}

En ce qui concerne les perceptions des acteurs scolaires vis-à-vis de l'intégration des TIC, l'exemple du PowerPoint a révélé les positions des apprenants et celles des enseignants. En effet, l'analyse est menée sous l'angle de l'apport de ce média aux apprentissages (figure 3), d'une part, et sous l'angle de son apport à l'acte d'enseignement, d'autre part (figure 4).

Les avantages du PowerPoint sont perçus par la majorité des directeurs régionaux et départementaux, en tant qu'apprenants en formation continue, comme un outil qui facilite la compréhension des cours du fait de leur clarté (58 \% des réponses); qui capte l'attention des apprenants (33\% des réponses); qui permet aux cours de finir dans le temps (17\% des réponses) et enfin un outil qui améliore les interactions entre le formateur et les auditeurs (cours dynamiques: $8 \%$ des réponses). Ces données de contexte issues des enquêtes corroborent une fois de plus l'apport positif des médias aux apprentissages.

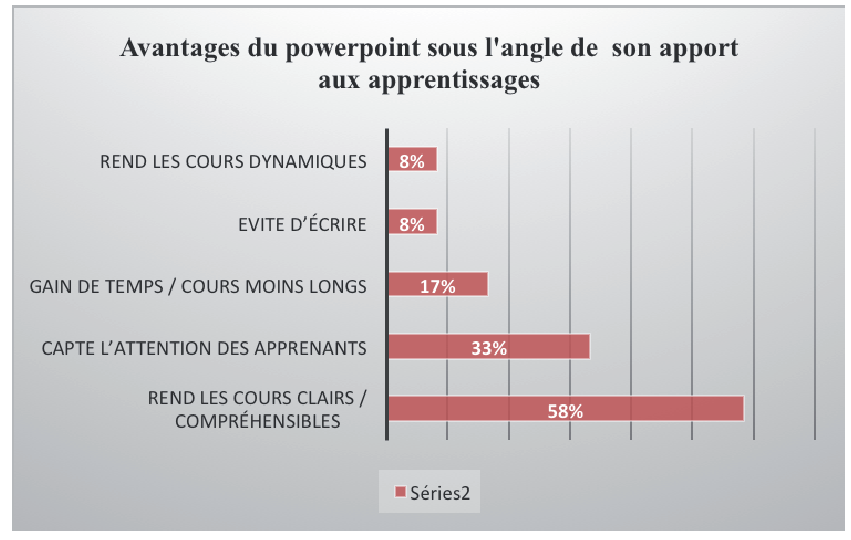

Figure 3

Les avantages de l'intégration des TIC sous l'angle de leur apport aux apprentissages 
Pour les enseignants responsables de la formation continue des personnels de l'éducation, le PowerPoint facilite l'acte d'enseignement, en ce sens que premièrement ce logiciel permet de synthétiser les cours et d'aller à l'essentiel dans leur préparation (67\% des réponses) ; par conséquent, les cours sont bien agencés (clarté des cours) et le temps pour les dispenser est par conséquent respecté (facilite une bonne gestion du temps). Deuxièmement, le PowerPoint permet le maintien de la motivation des apprenants du fait qu'il capte l'attention du début à la fin de l'exposé (58\% des suffrages), notamment du fait qu'il admet des illustrations. Troisièment, les diapositives soutiennent l'exposé du formateur (42\% des suffrages), ce qui lui permet de faire des cours moins magistraux, donc plus dynamiques (facilite les interactions enseignant-élèves).

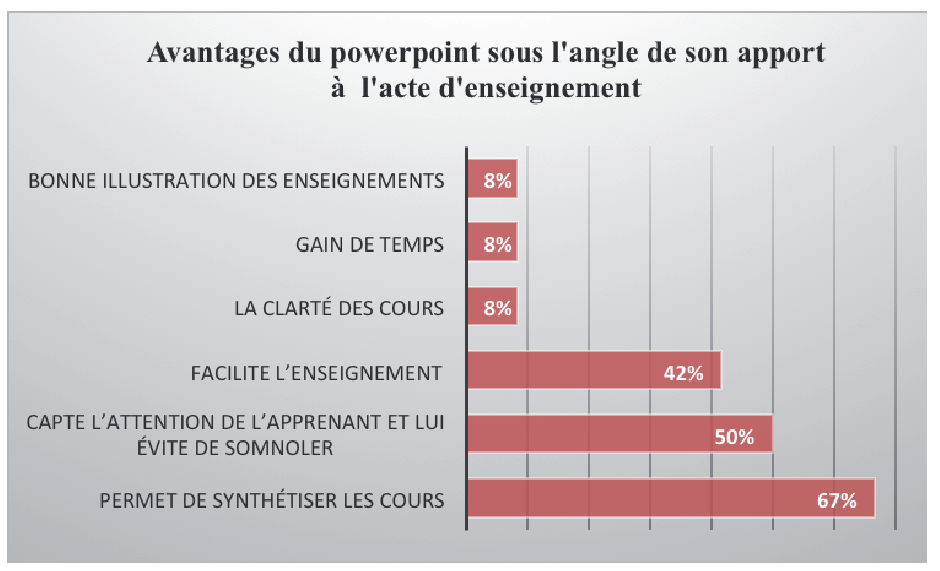

\section{Figure 4}

Les avantages de l'intégration des TIC sous l'angle de leur apport à l'acte d'enseignement

En somme, à travers les apports du PowerPoint dans les enseignements et dans les apprentissages, ce sont les potentialités pédagogiques des TIC qui sont ainsi mises en exergue. De plus, la formation à distance, en favorisant l'exploitation des outils informatiques aussi bien physiques que virtuels, permet aux usagers, enseignants et apprenants de se perfectionner dans l'utilisation des technologies éducatives.

Toutefois, les enquêtes ont révélé des appréhensions : celles-ci s'observent parmi les acteurs scolaires qui sont réfractaires vis-à-vis de la formation à distance, mais aussi parmi ceux qui y adhèrent.

\section{Analyse des appréhensions vis-à-vis de la formation à distance}

Seulement trois acteurs de la gouvernance éducative régionale sur les 12 interrogés, soit 16,66\%, désapprouvent ce mode pédagogique virtuel pour le renforcement de leurs capacités. Au titre des raisons avancées, ils estiment ne plus avoir le face-à-face avec le formateur, ce qui déshumanise à leur avis la formation. De plus, ils estiment que les enseignements en ligne ne permettront pas d'avoir les réponses en temps réel à leurs questions.

Pour l'ensemble des appréhensions exprimées en matière d'inconvénients (tableau 2) relevés au sujet de la formation à distance, l'absence de contact humain avec le formateur vient en tête avec $61,53 \%$ des suffrages, suivie des appréhensions concernant la couverture des directions régionales et 
départementales en internet et les aléas techniques tels que les problèmes de connexion et les pannes des machines (respectivement 15,38 \% des suffrages).

De l'analyse des raisons avancées, il ressort que les appréhensions des directeurs régionaux et départementaux sont en lien avec leur faible niveau de connaissance des possibilités pédagogiques des TIC. En effet, contrairement à leurs craintes, la formation en mode distanciel n'exclut pas les réponses aux questions en temps réel : c'est le cas des médias favorisant la communication synchrone.

\section{Tableau 2}

Inconvénients de la formation à distance selon les directeurs régionaux

\begin{tabular}{|c|c|c|}
\hline Principales avancées & $\begin{array}{l}\text { Suffrages } \\
\text { exprimés }\end{array}$ & Proportion (\%) \\
\hline $\begin{array}{l}\text { Absence de contact humain avec le formateur (on ne peut pas poser les questions } \\
\text { au formateur en temps réel) }\end{array}$ & 8 & 61,53 \\
\hline Insuffisance de la couverture en internet et les aléas techniques & 2 & 15,38 \\
\hline $\begin{array}{l}\text { Les aléas techniques pendant le cours (panne des machines et problème de connexion } \\
\text { internet) }\end{array}$ & 2 & 15,38 \\
\hline La tricherie favorisée (les apprenants peuvent faire faire les devoirs par une autre personne) & 1 & 7,69 \\
\hline $\begin{array}{l}\text { Total } \\
\end{array}$ & 13 & 100 \\
\hline
\end{tabular}

En ce qui a trait aux enseignants, les appréhensions (tableau 3) relevées quant à l'applicabilité de cette modalité de la formation continue sont d'abord relatives sur le plan de couverture des directions régionales et départementales en internet (50\% des réponses). Ensuite viennent les appréhensions relatives à l'absence de face-à-face avec l'apprenant qui peut être à leur avis, une source de démotivation $(35,71 \%)$ chez les apprenants. La non-maîtrise de l'usage des technologies éducatives par les formateurs est aussi une source d'appréhensions.

\section{Tableau 3}

Inconvénients de la formation à distance selon les enseignants

\begin{tabular}{l|l|l}
\hline Principales idées avancées & $\begin{array}{l}\text { Suffrages } \\
\text { exprimés }\end{array}$ & Proportion (\%) \\
\hline $\begin{array}{l}\text { Démotivation car on ne voit pas l'apprenant face-à-face / absence de relation directe } \\
\text { enseignant- élèves }\end{array}$ & 5 & 35,71 \\
\hline Faiblesse du débit de la connexion et de la couverture des régions en internet & 7 & 50,00 \\
\hline Coûts élevés des équipements & 1 & 7,14 \\
\hline Non-maîtrise de l'usage de l'outil informatique & 1 & 7,14 \\
\hline Total & $\mathbf{1 4}$ & $\mathbf{1 0 0}$ \\
\hline
\end{tabular}

En somme, pour la majorité des destinataires (animateurs et bénéficiaires) de l'innovation pédagogique à introduire, celle-ci a du sens. Cette signification que revêt la formation à distance pour ses destinataires est un indicateur d'acceptation des changements en perspective, des pratiques pédagogiques et de l'environnement d'apprentissage. 
Quant aux appréhensions sur le plan de la faisabilité de la formation à distance en matière d'équipement en matériels informatiques et de formation des destinataires en TIC, elles expriment la réalité des contraintes dans la mise en place de cette pratique pédagogique.

\section{Analyse des conditions de réalisation de la formation à distance}

L'analyse des conditions de réalisation de la formation à distance s'articule autour du niveau d'usage des technologies éducatives et d'équipement des organisations scolaires concernées par les enquêtes. Les suggestions faites révèlent aussi quelques contraintes.

\section{Le niveau de l'usage des technologies éducatives}

Le niveau de l'usage des technologies éducatives est évalué à travers le niveau de pratique des logiciels de mise en ligne de cours, de présentation d'exposés et de traitement de données.

Les logiciels les plus pratiqués par les futurs bénéficiaires de la formation à distance sont, par ordre de fréquence, PowerPoint (37,03 \%), Word (33,33 \%) et Excel (18,51\%), qui sont respectivement des logiciels de présentation d'exposés, de traitement de textes, de calculs et de construction de figures (tableau 4). La pratique du logiciel Moodle, incontournable à l'heure actuelle pour les enseignements en ligne, n'est pas encore effective parmi les directeurs régionaux et départementaux. Une formation à l'utilisation de cette plateforme numérique va donc constituer le préalable à l'intégration des cours à distance dans la formation continue.

\section{Tableau 4}

Les logiciels pratiqués par les directeurs régionaux et départementaux

\begin{tabular}{l|l|l|l}
\hline Logiciels pratiqués & Principales fonctions & $\begin{array}{l}\text { Suffrages } \\
\text { exprimés }\end{array}$ & Proportion (\%) \\
\hline Moodle & $\begin{array}{l}\text { Plateforme pédagogique (mise en ligne de cours } \\
\text { et autres ressources; communication synchrone et } \\
\text { asynchrone entre enseignant / élève) }\end{array}$ & 0 & 0,00 \\
\hline Zoom & Réunions, échanges synchrones et asynchrones & 2 & 7,40 \\
\hline PowerPoint & Présentation d'exposés (conférences et cours) & 10 & 37,03 \\
\hline Word initiation/intermédiaire & office, pour le traitement de textes & 9 & 33,33 \\
\hline Excel initiation/intermédiaire & office, pour les calculs et les graphiques & 5 & 18,51 \\
\hline Visioconférence & Exposés, conférences, cours à distance & 1 & 3,70 \\
\hline Total & & $\mathbf{2 7}$ & $\mathbf{1 0 0}$ \\
\hline
\end{tabular}

Les directeurs régionaux et départementaux ont été invités à estimer leur degré de maîtrise des technologies éducatives (tableau 5). En ce qui concerne la pratique des TIC physiques et virtuelles toutes confondues, la moitié des répondants $(50 \%)$ estiment qu'ils sont au degré de l'initiation et le quart d'entre eux, soit $25 \%$, se positionnent au degré intermédiaire 1 . Le faible degré de pratique des TIC constitue donc une autre contrainte dans la mise en œuvre de la formation à distance. 


\section{Tableau 5}

Degré de pratique des TIC parmi les directeurs régionaux et départementaux

\begin{tabular}{|l|l|l|}
\hline Degré de maîtrise des TIC & Nombre de cas & Proportion (\%) \\
\hline Degré initiation (de 0 à 20 sur une échelle de 0 à 100) & 6 & 50 \\
\hline Degré intermédiaire 1 (jusqu'à 40 sur une échelle de 0 à 100) & 3 & 25 \\
\hline Degré intermédiaire 2 (jusqu'à 60 sur une échelle de 0 à 100) & 2 & 17 \\
\hline Degré avancé (jusqu'à 80 sur une échelle de 0 à 100) & 0 & 0 \\
\hline Parfaite maîtrise (jusqu'à 100 sur une échelle de 0 à 100) & 1 & 8 \\
\hline Total des observations & $\mathbf{1 2}$ & $\mathbf{1 0 0}$ \\
\hline
\end{tabular}

Par comparaison, le degré de pratique des TIC parmi les enseignants est plus élevé que le degré de pratique parmi les directeurs régionaux, puisque la majorité d'entre eux se situent au niveau de pratique intermédiaire 2 (soit $43 \%$ d'entre eux ) et au niveau avancé (soit $25 \%$ d'entre eux) (tableau 6). Toutefois, cette légère avance ne doit pas occulter le fait que la maîtrise des TIC par les formateurs est un prérequis au moment de l'élaboration de la théorie du changement, donc en amont de celle des futurs apprenants.

\section{Tableau 6}

Degré de pratique des TIC parmi les enseignants

\begin{tabular}{|l|l|l|}
\hline Niveau de maîtrise des TIC par les formateurs & Nombre de cas & Proportion (\%) \\
\hline Degré initiation (de 0 à 20 sur une échelle de 0 à 100) & 2 & 16 \\
\hline Degré intermédiaire (jusqu’à 40 sur une échelle de 0 à 100) & 2 & 16 \\
\hline Degré intermédiaire 2 (jusqu'à 60 sur une échelle de 0 à 100) & 5 & 43 \\
\hline Degré avancé (jusqu'à 80 sur une échelle de 0 à 100) & 3 & 25 \\
\hline Parfaite maîtrise (jusquà 100 sur une échelle de 0 à 100) & 0 & 0 \\
\hline Total des observations & $\mathbf{1 2}$ & $\mathbf{1 0 0}$ \\
\hline
\end{tabular}

De toute façon, que l'on soit enseignant ou apprenant, la pratique des outils informatiques est un prérequis pour les formations en ligne.

Le niveau d'équipement des directions régionales et de l'institution supérieure de formation des personnels de l'Enseignement Technique

L'insuffisance des équipements informatiques dans le contexte scolaire ivoirien représente une contrainte majeure dans la mise en oeuvre de la formation à distance (tableau 7). En effet, la moitié des acteurs de la gouvernance scolaire régionale estiment que le niveau d'équipement de leurs lieux de service en matériels informatiques se situe entre 0 et 25 sur une échelle de 0 à 100 . Sur cette échelle de mesure, au total $75 \%$, soit les trois quarts des directions régionales et départementales de l'étude, ont un niveau d'équipement en dessous de la moyenne. 


\section{Tableau 7}

Niveau d'équipement des directions régionales et départementales

\begin{tabular}{|l|l|l|}
\hline $\begin{array}{l}\text { Niveau d'équipement des directions régionales } \\
\text { (Sur une échelle de 0 à 100) }\end{array}$ & Nombre de cas & Proportion (\%) \\
\hline $0-25$ & 6 & 50 \\
\hline $25-50$ & 3 & 25 \\
\hline $50-75$ & 1 & 8 \\
\hline $75-100$ & 2 & 17 \\
\hline Total des observations & $\mathbf{1 2}$ & $\mathbf{1 0 0}$ \\
\hline
\end{tabular}

La totalité des enseigants interrogés possède un ordinateur portable ; toutefois, le ratio moyen de 4 enseignants pour 1 vidéoprojecteur, soit un taux de couverture de $33 \%$, limite les possibilités de pratiquer régulièrement les enseignements hybrides avec le PowerPoint, pour tous les formateurs (tableau 8). Un constat positif est fait cependant en faveur de l'intégration de nouveaux outils informatiques dans les enseignements, avec l'exploitation récente de la plateforme pédagogique Moodle. En effet, la pandémie du coronavirus a été l'environnement déclencheur et $75 \%$ des enseignants interrogés dans cette enquête s'en servent depuis peu, pour terminer le programme de formation. Ainsi, les logiciels Moodle et Zoom, du fait de cette situation de pandémie, sont en train d'entrer dans les pratiques pédagogiques des enseignants de cette institution de formation.

\section{Tableau 8}

Niveau d'équipement de l'institution de formation des personnels de l'Enseignement Technique

\begin{tabular}{|l|l|l|}
\hline Type d'équipement & Niveau d'équipement & Proportion (\%) \\
\hline Ordinateur portable & 12 enseignants /12 interrogés & 100 \\
\hline Ratio (enseignant / rétroprojecteur) & 4 enseignants /1 rétro- projecteur & 33 \\
\hline Tableau à écran interactif & 2 salles équipées / 16 & 12,5 \\
\hline Exploitation plateforme Moodle & 9 enseignants/12 interrogés & 75 \\
\hline
\end{tabular}

Le contexte mondial actuel de pandémie est donc un facteur facilitant pour l'intégration des outils numériques dans les activités pédagogiques. Les suggestions pour la planification de la mise en œuvre de la formation à distance en sont l'illustration.

\section{Les suggestions pour la planification de la mise en ouvre de la formation à distance}

Les suggestions des acteurs en présence sont regroupées en deux catégories : celles exprimées en matière de besoins de formation et celles exprimées pour un meilleur équipement des structures de formation concernées. 
De façon majoritaire, les suggestions des directeurs régionaux et départementaux concernent leurs besoins de formation en TIC (tableau 9). Cette formation constitue un prérequis pour le renforcement de leurs capacités en gouvernance scolaire par le mode distanciel.

\section{Tableau 9}

Les suggestions des directeurs régionaux et départementaux

\begin{tabular}{|l|l|l|}
\hline Suggestions principales & $\begin{array}{l}\text { Suffrages } \\
\text { exprimés }\end{array}$ & Proportions (\%) \\
\hline $\begin{array}{l}\text { Suggestions en matière de besoins de formation } \\
\text { Être formé aux nouvelles méthodes de management des écoles, avec des contenus } \\
\text { professionnalisants; être formé en TIC - Notamment en Word, Excel, PowerPoint, } \\
\text { Moodle, Zoom - ;être formé en technique d'expression et en culture générale). }\end{array}$ & 8 & 50 \\
\hline $\begin{array}{l}\text { Suggestions pour un meilleur équipement en ressources informatiques } \\
\text { Avoir une connexion internet haut débit ; doter les établissements de salles adaptées } \\
\text { (salles de conférence et salles de cours avec équipements de pointe); nombre suffisant } \\
\text { d'ordinateurs. }\end{array}$ & 5 & 31,25 \\
\hline Autres types de suggestions & 3 & 18,75 \\
\hline Total des suffrages & $\mathbf{1 6}$ & $\mathbf{1 0 0}$ \\
\hline
\end{tabular}

Contrairement aux directeurs régionaux, les suggestions des enseignants visent davantage un meilleur équipement en matériels informatiques des organisations scolaires, pour une connexion internet de qualité (tableau 10). 


\section{Tableau 10}

Les suggestions des enseignants responsables de la formation continue

\begin{tabular}{|l|l|l|}
\hline Suggestions principales & $\begin{array}{l}\text { Suffrages } \\
\text { exprimés }\end{array}$ & Proportions (\%) \\
\hline $\begin{array}{l}\text { Suggestions pour un meilleur équipement en ressources informatiques } \\
\text { Avoir internet haut débit et accessible à tout le personnel enseignant ; avoir les } \\
\text { équipements requis pour les cours (vidéo projecteurs, ordinateurs, tableaux à écrans } \\
\text { interactifs, des logiciels...). }\end{array}$ & 10 & 62,5 \\
\hline $\begin{array}{l}\text { Suggestions en matière de besoins de formation } \\
\text { Formation des enseignants aux logiciels de cours en ligne ; inclure un module de } \\
\text { formation en TIC pour les apprenants ; faire suivre ces cours de stages pratiques de prise } \\
\text { en mains des outils informatiques. }\end{array}$ & 6 & 37,5 \\
\hline Total des suffrages & $\mathbf{1 6}$ & $\mathbf{1 0 0}$ \\
\hline
\end{tabular}

L'analyse globale des suggestions révèle que l'intégration des enseignements en ligne dans la formation continue est liée aux conditions de sa mise en œuvre peu propices. En effet, la reconnaissance de la pression au changement est indissociable des conditions concrètes d'application de ce changement.

\section{Discussion des résultats}

Il ressort des résultats que la formation à distance est perçue comme une alternative intéressante à la formation continue classique en mode présentiel. En effet, l'analyse des approbations exprimées a révélé la trilogie : mise à jour des connaissances et des pratiques de gestion; annulation des coûts inhérents au déplacement et le gain de temps. Cette large adhésion pourrait s'expliquer par le sens que revêt cette innovation pédagogique pour les acteurs en présence. Crozier et Friedberg (1997) expliquent à ce sujet que les personnes changent ou sont motivées à changer que si elles s'approprient concrètement le changement et en perçoivent les enjeux. De même, Gagnon (2013) cité par Bilodeau (2017, p. 35 à p. 38) explique qu'un savoir est signifiant pour un individu dans la mesure où il est pertinent pour celui-ci et valide à ses propres yeux, conformément à sa position épistémologique.

Par ailleurs, les effets positifs de l'usage des TIC ont été reconnus à travers les opinions sur l'apport du PowerPoint aux apprentissages et à l'acte d'enseignement.

Les avantages du PowerPoint sont perçus par la majorité des directeurs régionaux et départementaux, en tant qu'apprenants en formation continue, comme un outil qui facilite la compréhension des cours du fait de leur clarté (58 \% des réponses); qui capte l'attention des apprenants (33\% des réponses); qui permet aux cours de finir dans le temps (17\% des réponses) et enfin un outil qui améliore les interactions entre le formateur et les auditeurs (cours dynamiques: $8 \%$ des réponses). p.10 de cet article. 
Pour sa part, Clark (1994) apporte une nuance à cette position, en affirmant que l'influence des technologies sur l'apprentissage est nulle dans la mesure où la qualité de l'apprentissage est liée à l'intégration du processus d'instruction dans le média. C'est donc la qualité de l'exploitation pédagogique du média qui fait l'efficacité de l'enseignement avec les TIC. Le média à lui seul ne peut garantir la réussite d'un acte pédagogique ; toutefois, il peut l'amplifier. La technologie éducative, en se servant d'une théorie de l'apprentissage, crée les conditions nécessaires à l'émergence d'un apprentissage. Les technologies sous différentes formes ont donc des effets positifs sur les apprentissages quand elles sont exploitées adéquatement.

Quant aux appréhensions exprimées vis-à-vis de la formation à distance, leur analyse a révélé deux craintes principales : l'absence d'interactions entre apprenants et enseignants et la faiblesse du débit de la connexion internet du fait de l'équipement insuffisant des structures scolaires. À propos des appréhensions selon lesquelles la formation en ligne déshumanise la classe et ne permet pas des interactions en temps réel, elles sont à mettre sur le compte de la méconnaissance des possibilités de communications synchrones dans les enseignements en ligne (Karsenti, 2019). En effet, des logiciels de réunions tels que Zom (appels-conférence) et le Chat permettent des échanges en temps réel, non seulement entre enseignants et apprenants, mais aussi entre apprenants dans le cadre du travail en équipe.

Par ailleurs, selon certains répondants lors des enquêtes, la formation à distance favoriserait les cours magistraux et les méthodes non actives. Or, Clark (1994) cité par Fenouillet et Déro (2006) explique qu'une telle perception revient à confondre moyens pédagogiques et approche pédagogique. En fait, c'est une technologie donnée qui se sert d'une théorie de l'apprentissage pour créer les conditions nécessaires à l'émergence d'un apprentissage. Par exemple, l'approche pédagogique par les compétences, qui met en œuvre le constructivisme, est aussi bien pratiquée en mode présentiel qu'à distance. Les cours en ligne mettent l'apprenant dans une position où il est face à l'objet de son apprentissage sans une intervention dirigiste de l'enseignant. Cette situation d'apprentissage l'incite à construire son savoir avec l'enseignant comme guide. De plus, au regard des ressources variées proposées aux étudiants, par exemple dans le cas de la plateforme Moodle qui comporte une interface favorisant l'exploitation de multiples ressources et activités, les appréhensions des directeurs régionaux et départementaux à subir des cours avec des méthodes pédagogiques non actives ne sont pas fondées. Toutefois, Hodges et Repman (2011) font remarquer que les plateformes pédagogiques sont majoritairement utilisées par les enseignants pour uniquement déposer des supports et des exercices, et elles sont exploitées par les étudiants, juste pour télécharger les cours déposés ou pour la remise des devoirs. Ces auteurs concluent donc que l'environnement de formation constaté actuellement est calqué sur le modèle transmissif plutôt que sur le modèle constructiviste. Cette situation, à notre sens, est liée à la non-maîtrise de l'usage de la plateforme Moodle et des outils de communication synchrone par les enseignants, au regard du niveau peu avancé de la majorité d'entre eux dans la pratique des technologies éducatives. C'est ce qui explique la persistance du modèle transmissif dans les pratiques actuelles sur les plateformes pédagogiques et la quasi-absence constatée du modèle constructiviste.

Quant aux appréhensions exprimées, Jodelet (2006) les explique par le fait que les gens sont d'accord pour poursuivre une action de changement dans ce qu'elles ont déjà l'habitude de réussir, du moins partiellement. Ainsi, la formation à distance ne remplacera pas la formation en mode présentiel, mais 
elle viendra progressivement en complément, pour une formation continue efficace des personnels de l'éducation.

Par ailleurs, à côté des appréhensions exprimées, l'insuffisance de l'équipement en matériels informatiques des organisations scolaires et l'usage limité des technologies éducatives par les acteurs scolaires sont à relever au titre des contraintes. Cette position du présent article est corroborée par Karsenti (2019) qui, dans une méta-analyse, a trouvé que le plus important potentiel des technologies est atteint lorsque chaque apprenant possède son propre outil informatique, lorsqu'il est lui-même formé aux usages éducatifs des technologies, et aussi lorsque son enseignant a développé les compétences nécessaires lui permettant de mieux enseigner avec le numérique. Les conditions de réalisation de la formation à distance sont d'autant plus importantes que la reconnaissance de la pression au changement est indissociable des conditions concrètes d'application de ce changement.

\section{Conclusion}

La mise en ligne des cours représente désormais un enjeu en matière de performance du système éducatif, au regard de la relation entre la qualité des services éducatifs et le renforcement régulier des capacités des ressources humaines. La présente étude a ainsi analysé les avantages et les contraintes de la formation à distance, à travers les approbations, appréhensions et suggestions des directeurs régionaux et départementaux de l'Enseignement technique en Côte d'Ivoire et celles des enseignants chargés du renforcement de leurs capacités. Les enquêtes ont révélé une adhésion de principe des acteurs concernés, à la formation à distance comme une alternative au renforcement de leurs capacités en mode présentiel. Toutefois, un travail d'accompagnement de l'appropriation de cette innovation pédagogique reste à faire, au regard des contraintes répertoriées. Ces contraintes se déclinent en matière d'insuffisance d'équipements en matériels informatiques, d'usage limité des technologies éducatives parmi les acteurs scolaires et d'appréhensions de ces acteurs face au changement d'habitudes professionnelles en perspective. Cette étude qui épouse le paradigme de la recherche collaborative a impliqué les futurs bénéficiaires et animateurs de la formation à distance, dans la réflexion sur ses avantages éducatifs et les contraintes de sa mise en œuvre. C'est un partage réflexif entre le milieu de la recherche et le milieu de la pratique. Cet article veut ainsi contribuer, à travers des données empiriques, à faire de ce changement intentionnel consigné dans le plan stratégique de la réforme de l'Enseignement technique 2012-2021, un changement émergent. De plus, le contexte de pandémie qui prévaut actuellement constitue, à notre sens, un environnement facilitant pour que les conditions matérielles d'exploitation de la formation à distance comme un complément de l'enseignement en mode présentiel soient notablement améliorées dans le système éducatif. Un nouvel environnement d'apprentissage apparaît en effet, avec le recours aux enseignements en ligne pour terminer les programmes de formation.

\section{Note}

1 http://stms-bse.discipline.ac-lille.fr/de-lusage-du-numerique/les-outils/logiciels-denquete/tutoriel-sphinx 


\section{Références bibliographiques}

Bedin, V. et Fournier, M. (2014). Psychologie des apprentissages. Dans V. Bedin et M. Fournier Apprendre : pourquoi et comment? (pé 7-12). Sciences Humaines.

Bilodeau, K., (2017). Pratiques pédagogiques et développement de savoirs signifiants en formation à l'exercice d'un métier semi-spécialisé, Thèse de doctorat, Université Laval.

Clark, R.E. (1994a). Media will never influence learning. Educational technology research and development, 42 (2), 21-29.

Crozier, M. et Friedberg, E. (1981) l'acteur et le système : les contraintes de l'action collective, Editions du Seuil, 1981, P. 202. Première parution en 1977, dans la collection « Sociologie politique »

Deschênes, Bilodeau, Bourdages, Dionne, Gagné, Lebel et Rada-Donat (1996). Constructivisme et formation à distance, in DistanceS, vol. 1, numéro 1, printemps 1996, p. 9 à 21, source internet : http://cqfd.teluq.uquebec.ca/Activites/DistanceS/dist ant/vol1N1/vol1n1.html

Deschênes, A.-J. et Lebel, C. (1994). La conception du support à l'apprentissage dans des activités de formation à distance. La formation à distance maintenant (Thème II, Texte IV). Télé-université.

Deschênes, A.J et Maltais, M. (2016). Formation à distance et accessibilité, téléuniversité, 2006, edutice-00078809.

Fenouillet, F., Déro, M., (2006). Le e-learning est-il efficace ? Une analyse de la littérature anglo-saxonne. Savoirs, 3(12), 88-101.

Gagnon, R. (2013). Eduquer après Carl Gustav Jung suivi de Métaphores et autres vérités,

Université Laval.

Hodges, C. et Repman, J. (2011). Moving outside the LMS : Matching web2.0 tools to instructional purpose. EDUCAUSE Learning Initiative, september 7, 2011.

Jodelet, D. (2006). Représentation sociale. Dans S. Mesure, P. Savidan (dir.), Le dictionnaire des sciences humaines (p. 10031005). PUF.

Karsenti,T. (2019). «Impacts de l'utilisation des outils numériques en éducation », Rapport : Utilisation du numérique dans les écoles, Abidjan, 7 juillet 2019, p.3.

Legendre-Bergeron, M.-F. (1980). Lexique de la psychologie du développement de Jean Piaget. Gaëtan Morin.

\section{Pour citer cet article}

Aye, M-F, A. (2020). Avantages et contraintes de la formation à distance des acteurs de la gouvernance scolaire régionale en Côte d'Ivoire. Formation et profession, 28(4 hors-série), 1-16. http://dx.doi.org/10.18162/fp.2020.700 